\title{
Incidence of Second Malignancy in Patients with Papillary Thyroid Cancer from Surveillance, Epidemiology, and End Results 13 Dataset
}

\author{
Mayumi Endo ${ }^{1},{ }^{1,2}$ Jessica B. Liu, ${ }^{3}$ Marcelle Dougan, ${ }^{4}$ and Jennifer S. Lee ${ }^{2}$ \\ ${ }^{1}$ Division of Endocrinology, Diabetes and Metabolism, Department of Internal Medicine, Ohio State University School of Medicine, \\ 578 McCampbell Hall, 1581 Dodd Drive, Columbus, OH 43210, USA \\ ${ }^{2}$ Division of Endocrinology, Gerontology and Metabolism, Department of Internal Medicine, Stanford University School of Medicine, \\ 300 Pasteur Drive, Grant S-025, Stanford, CA 94305, USA \\ ${ }^{3}$ Perinatal Epidemiology and Health Outcomes Research Unit, Division of Neonatology, Department of Pediatrics, \\ Stanford University School of Medicine and Lucile Packard Children's Hospital, 1265 Welch Road, Stanford, CA 94305, USA \\ ${ }^{4}$ Department of Health Science and Recreation, San Jose State University, One Washington Square, San Jose, CA 95192, USA
}

Correspondence should be addressed to Mayumi Endo; mayumi.endo@osumc.edu

Received 18 January 2018; Accepted 14 March 2018; Published 26 June 2018

Academic Editor: Noriyuki Koibuchi

Copyright (c) 2018 Mayumi Endo et al. This is an open access article distributed under the Creative Commons Attribution License, which permits unrestricted use, distribution, and reproduction in any medium, provided the original work is properly cited.

Increased risk of second primary malignancy (SPM) in papillary thyroid cancer (PTC) has been reported. Here, we present the most updated incidence rates of second primary malignancy from original diagnosis of PTC by using the data from the Surveillance, Epidemiology, and End Results. In this cohort, 3,200 patients developed SPM, a substantially higher number than in the reference population of 2,749 with observed to expected ratio (O/E) of 1.16 (95\% CI; 1.12-1.21). Bone and joint cancer had the highest O/E ratio of 4.26 (95\% confidence interval [CI] 2.33-7.15) followed by salivary gland (O/E 4.15; 95\% CI 2.76-6.0) and acute lymphocytic leukemia (O/E 3.98; 95\% CI 2.12-6.8). Mean age at the diagnosis of SPM was 64.4 years old. Interestingly, incidence of colorectal cancer was lower in thyroid cancer survivors compared to general population (large intestine O/E 0.3; 95\% CI 0.06-0.88, rectum O/E 0.6; 95\% CI 0.41-0.85); however, this was not observed in patients who underwent radiation therapy. The incidence of SPM at all sites was higher during 2000-2012 compared to 1992-1999 (O/E 1.24 versus 1.10). Surprisingly, patients with micropapillary cancer had higher incidence of SPM than counterparts with a larger tumor in radiation group (O/E of 1.40 versus 1.15). O/E of all cancers were higher in males compared to females with O/E of 1.41 versus 1.17 during the period of 2000-2012. Diagnosis of PTC before age 50, especially at age 30-34, was associated with higher incidence of overall SPM (age 30-34; O/E 1.43; 95\% CI; 1.19-1.71). Efficient monitoring strategies that include age at the time of thyroid cancer diagnosis, exposure to radiation, gender, and genetic susceptibility may successfully detect SPM earlier in the disease course. This is especially important given the excellent prognosis of the initial thyroid cancer itself.

\section{Introduction}

Papillary thyroid cancer (PTC) is rapidly increasing both in the United States and abroad $[1,2]$. Since 1975, the incidence of PTC has nearly tripled, from 4.9 to 14.3 per 100,000 individuals (absolute increase, 9.4 per 100,000; relative rate [RR], 2.9; 95\% CI, 2.7-3.1) based on data from the Surveillance, Epidemiology, and End Results (SEER) dataset [1]. Given the dramatic increases in disease prevalence and a high five-year survival rate of more than $95 \%$ [3], monitoring of long-term treatment outcomes and side effects after initial treatment is important.

Increased risk of second primary malignancy (SPM) in PTC has been reported in several cancer registry and epidemiologic studies [3-10]. It is hypothesized that increased risk of SPM may be related to a genetic predisposition or treatment-related complication. Radioactive iodine therapy (RAI), which has been a common adjuvant therapy for the management of PTC, typically following surgery, has been a target of debate due to side effects such as sialadenitis, 


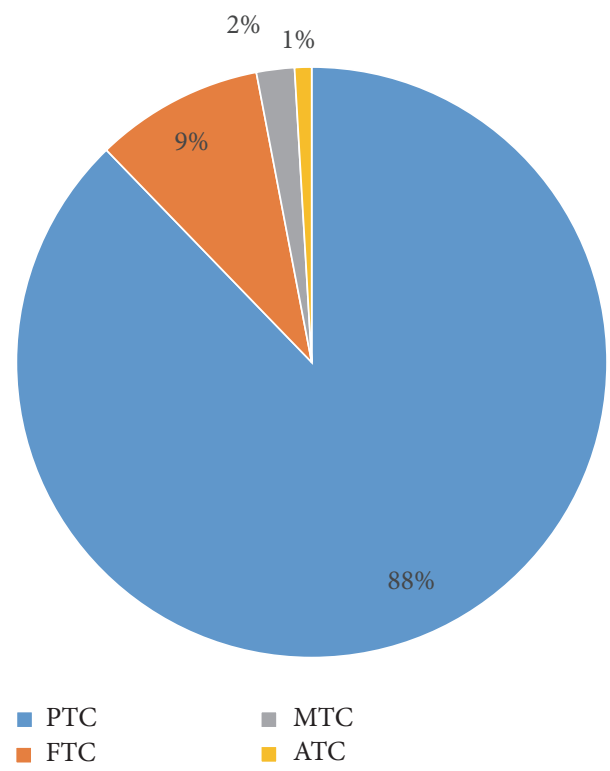

FIgure 1: Prevalence of Papillary Thyroid Cancer in SEER 13 cohort. PTC: papillary thyroid cancer, FTC: follicular thyroid cancer, MTC: medullary thyroid cancer, and ATC: anaplastic thyroid cancer.

taste loss, and, most critically, SPM. Numerous cancers are thought to be induced from radiation exposure, based on epidemiologic studies involving environmental, medical, and occupational exposures [11-23]. Using a SEER 9 database consisting of 52,103 patients, Kim et al. demonstrated that salivary cancer, kidney cancer, breast cancer, prostate cancer, melanoma, non-Hodgkin lymphoma, leukemia, multiple myeloma, brain cancer, and thyroid cancer were increased in patients with history of PTC and RAI, compared to those without a history of RAI [3].

Here, we present updated incidence rates of SPM after PTC using SEER 13 data.

\section{Materials and Methods}

2.1. Study Population. The study population was assembled using records from the SEER program of the National Cancer Institute. A $98 \%$ case ascertainment is mandated from 14 population-based registries and three supplemental registries representing approximately $26 \%$ of the US population [9]. In particular, our cohort from the SEER 13 registries consists of data from Atlanta, Connecticut, Detroit, Hawaii, Iowa, New Mexico, San Francisco-Oakland, Seattle-Puget Sound, Utah, Los Angeles, San Jose-Monterey, Rural Georgia, and the Alaska Native Tumor Registry. Data are available for people with cancer diagnosed from 1973 and later, with the exception of Seattle-Puget Sound, Atlanta Los Angeles, San Jose-Monterey, Rural Georgia, and the Alaska Native Tumor Registry. The Seattle-Puget Sound and Atlanta registries joined the SEER program in 1974 and 1975 and Los Angeles, San Jose-Monterey, Rural Georgia, and the Alaska Native Tumor Registry joined in 1992, respectively. The SEER registries contain information on patient demographics, tumor site, histology, date and source of diagnosis, lymph node and distant metastasis status, extrathyroidal extension, multifocality (recorded since 2004), date of death, and treatment. The SEER program classifies patients as N0 based on pathologic analysis or on clinical and radiographic data if patients do not undergo lymph node dissection. Each year, quality and completeness studies are conducted in SEER areas to ensure high quality data. The baseline cohort for this analysis consisted of individuals diagnosed with a primary thyroid cancer and identified by site code ICD-0-3:C739, reported to SEER 13 database between 1992 and $2013(n=75,992)$. Males and females of all ages and US Office of Management and Budget race/ethnicity codes (OMB codes) were included in this analysis. We limited tumor histology to PTC, which consists of $88 \%$ of all thyroid cancers (Figure 1), by limiting our histology code to M8050, M8340-8344, and M8450. In addition, several stratified analyses were conducted by various characteristics of the first thyroid cancer, which included tumor size $(0-10 \mathrm{~mm}, 11-20 \mathrm{~mm}, 21-50 \mathrm{~mm}$, and $>51 \mathrm{~mm})$, year of the diagnosis of the thyroid cancer, and the status of radiation treatment (categorized by no radiation, isotopes only, beam radiation, and radiation not otherwise specified). Any SPM within the first 6 months after initial thyroid cancer was excluded. SPMs were classified according to Standard Warren and Gates criteria modified by the NCI (REF).

2.2. Statistical Analysis. The number of observed SPM was determined from the SEER 13 database. Expected cancers were calculated based on the 2000 US standard population distribution. The risk of SPM was defined as the standardized incidence ratio (SIR) adapted for cancer registry analysis $[24,25]$. The SIR is the ratio of observed to expected $(\mathrm{O} / \mathrm{E})$ second cancers, in which the expected number is calculated for a reference cohort of identical age, gender, race, and time. Risks of SPM were stratified by gender and age at the time of the initial thyroid cancer diagnosis, time since diagnosis, and type of treatment (radioisotope therapy, beam radiation, and no radiation). Confidence intervals (CI) and $p$ values were at 0.05 significance alpha levels and two-sided based on Poisson exact methods. To avoid statistically unstable estimates, SIRs and CI were not presented where the number of observed cancers was less than five. The excess risk was determined by subtracting the expected number from the observed number of second cancers and then dividing the difference by the number of person at risk. All analyses were conducted with statistical program SEER $*$ Stat version 8.3.5 provided by the National Cancer Institute utilizing the multiple primary standardized incidence ratio (MP-SIR) tool.

\section{Results}

3.1. Elevated Risk of SPM in PTC Cases. In this cohort, 3,200 patients developed SPM (Table 1), a substantially higher number than in the reference population of 2647 (Table 1). Of these, 2161 were female (67.5\%) and 1,039 (32.5\%) were male. Incidence of SPM increased over time in both females and males; incidence was higher during 2000-2012 (O/E in male 1.41, O/E in female 1.17) compared 1992-1999 (O/E in male 1.16, $\mathrm{O} / \mathrm{E}$ in female 1.08). Bone and joints cancer had the highest $\mathrm{O} / \mathrm{E}$ ratio of 4.26 (95\% confidence interval 
TABLE 1: Prevalence of primary second malignancy in papillary thyroid cancer survivors, SEER 13 cohort.

\begin{tabular}{|c|c|c|c|c|c|c|}
\hline & Observed & Expected & $\mathrm{O} / \mathrm{E}$ & $95 \% \mathrm{CI}$ & Excess risk & Mean age at event \\
\hline All sites & 3,200 & $2,749.03$ & $1.16 \#$ & $1.12-1.21$ & 13.15 & 64.45 \\
\hline All solid tumors & 2,839 & $2,464.99$ & $1.15 \#$ & $1.11-1.19$ & 10.91 & 64.38 \\
\hline Salivary Gland & 28 & 6.74 & $4.15 \#$ & $2.76-6.0$ & 0.62 & 56.91 \\
\hline Large intestine & 3 & 9.98 & $0.30 \#$ & $0.06-0.88$ & -0.2 & 75.42 \\
\hline Rectum & 32 & 52.95 & $0.60 \#$ & $0.41-0.85$ & -0.61 & 60.12 \\
\hline $\begin{array}{l}\text { Rectum, rectosigmoid junction, } \\
\text { anus, anal canal and anorectum }\end{array}$ & 63 & 83.41 & $0.76 \#$ & $0.58-0.97$ & -0.6 & 62.89 \\
\hline Bones and joints & 14 & 3.29 & $4.26 \#$ & $2.33-7.15$ & 0.31 & 58.44 \\
\hline Soft tissue including heart & 31 & 15.93 & $1.95 \#$ & $1.32-2.76$ & 0.44 & 60.84 \\
\hline $\begin{array}{l}\text { Skin excluding basal and } \\
\text { squamous }\end{array}$ & 182 & 129.47 & $1.41 \#$ & $1.21-1.63$ & 1.53 & 61.53 \\
\hline Melanoma of the skin & 162 & 118.65 & $1.37 \#$ & $1.16-1.59$ & 1.26 & 61.02 \\
\hline Breast & 727 & 621.47 & $1.17 \#$ & $1.09-1.26$ & 3.08 & 61.06 \\
\hline Prostate & 316 & 259.04 & $1.22 \#$ & $1.09-1.36$ & 1.66 & 67.38 \\
\hline Urinary bladder & 129 & 99.46 & $1.30 \#$ & $1.08-1.54$ & 0.86 & 71.92 \\
\hline Kidney & 152 & 70.32 & $2.16 \#$ & $1.83-2.53$ & 2.38 & 63.68 \\
\hline Ureter & 8 & 2.94 & $2.72 \#$ & $1.17-5.36$ & 0.15 & 74.16 \\
\hline $\begin{array}{l}\text { All lymphatic and hematopoietic } \\
\text { diseases }\end{array}$ & 294 & 224.83 & $1.31 \#$ & $1.16-1.47$ & 2.02 & 64.78 \\
\hline Lymphoma & 147 & 122.31 & $1.20 \#$ & $1.02-1.41$ & 0.72 & 64.15 \\
\hline Hodgkin lymphoma & 11 & 9.61 & 1.14 & NS & 0.04 & 38.58 \\
\hline Non-Hodgkin lymphoma & 136 & 112.69 & $1.21 \#$ & $1.01-1.43$ & 0.68 & 66.22 \\
\hline Myeloma & 44 & 35.26 & 1.25 & NS & 0.25 & 71.5 \\
\hline Leukemia & 103 & 67.26 & $1.53 \#$ & $1.25-1.86$ & 1.04 & 62.81 \\
\hline Lymphocytic leukemia & 45 & 32.74 & $1.37 \#$ & $1-1.84$ & 0.36 & 63.81 \\
\hline Acute lymphocytic leukemia & 13 & 3.27 & $3.98 \#$ & $2.12-6.8$ & 0.28 & 58.2 \\
\hline Chronic lymphocytic leukemia & 32 & 27.42 & 1.17 & NS & 0.13 & 66.09 \\
\hline Nonlymphocytic leukemia & 58 & 34.52 & $1.68 \#$ & $1.28-2.17$ & 0.68 & 62.04 \\
\hline $\begin{array}{l}\text { Acute nonlymphocytic leukemia } \\
\text { (ANLL) }\end{array}$ & 40 & 23.15 & $1.73 \#$ & $1.23-2.35$ & 0.49 & 63.89 \\
\hline Myeloid and monocytic leukemia & 55 & 31.41 & $1.75 \#$ & $1.32-2.28$ & 0.69 & 61.09 \\
\hline Acute myeloid leukemia & 38 & 20.59 & $1.85 \#$ & $1.31-2.53$ & 0.51 & 65.06 \\
\hline
\end{tabular}

Cancers without statistically significant elevated risk are excluded, except for ones related to other statistically significant cancers; \#: $p$ value $<0.05$; $95 \%$ CI: 95\% confidence interval; NS: statistically not significant.

[CI] 2.33-7.15) followed by salivary gland (O/E 4.15; 5\% CI 2.76-6.0), acute lymphocytic leukemia (O/E; 3.98, 95\% CI 2.12-6.8), and ureter cancer (O/E $2.7295 \%$ CI 1.17-5.36). Mean age at the diagnosis of SPM was 64.4 years old. Interestingly, thyroid cancer survivors had a decreased risk of the development of colorectal cancer compared to reference population (large intestine $\mathrm{O} / \mathrm{E} 0.3$; $95 \% \mathrm{CI} 0.06-0.88$, rectum O/E 0.6; 95\% CI 0.41-0.85).

3.2. Elevated SPM Risks in Patients Who Underwent Radiation Therapy. Patients who had a radioisotope therapy had the higher O/E of overall SPM (O/E 1.16; 95\% CI 1.1-1.23)
(Table 2) compared to nonradiation group (O/E 1.09; 95\% CI, 1.03-1.14). Beam radiation group did not have significant increase in overall SPM, possibly due to small observed number of 82 cases. There were two cancers which showed marked increase in radioisotope group compared to nonradiation group; salivary gland cancer $(\mathrm{O} / \mathrm{E} 7.8$ versus 1.78$)$ and leukemia (O/E 2.20 versus 1.05$)$ were with the highest $\mathrm{O} / \mathrm{E}$ observed in chronic myeloid leukemia (O/E 2.92; 95\% CI 1.34-12.93). Nonradiation group had a decrease in risk of colorectal cancer but this was not replicated in radiation group. Instead, beam radiation group had an increased risk of colon cancer (O/E 2.0; 95\% CI 1.07-3.63). Incidence of 


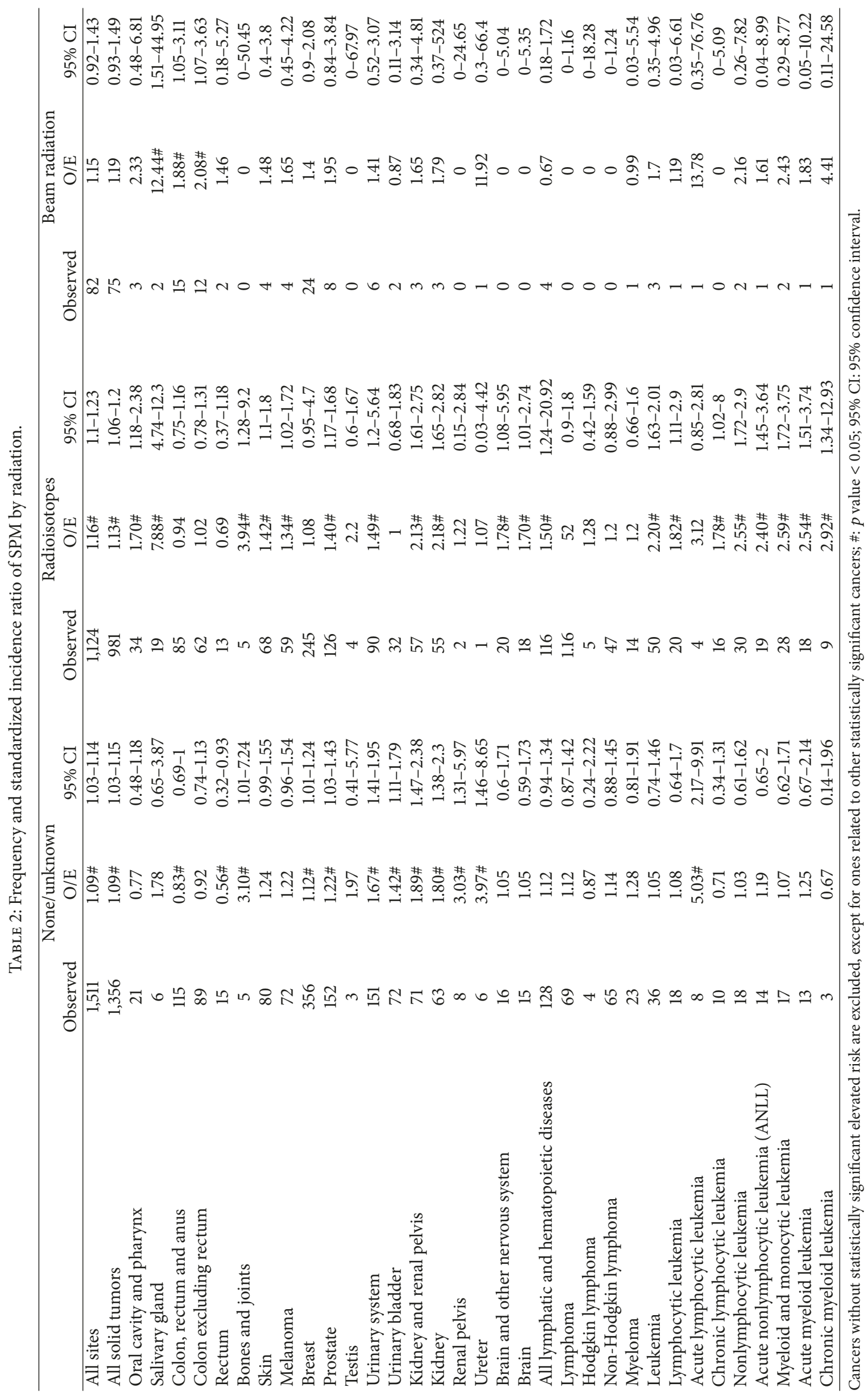


bone and joint cancer, kidney cancer, and prostate cancer was elevated both in nonradiation and in radioisotope group with higher $\mathrm{O} / \mathrm{E}$ in radioisotope group. There were 26 patients who received both radioisotope and radiation therapy and subsequently developed SPM. There was no statistically significant increase in SPM compared to reference population for this group (data not shown).

3.3. Elevated SPM Risk in Patients with Micropapillary Cancer. Patients with micropapillary cancer (MPTC), defined as tumor diameter of less than $1 \mathrm{~cm}$ at largest diameter, had higher incidence of SPM than counterparts with a larger tumor, particularly in patients who underwent radiation therapy (Table 3). O/E of SPM at all sites in patients without radiation was 1.21 in MPTC whereas O/E of PTC above $1 \mathrm{~cm}$ was 1.04. In the radiation group, MPTC had a higher O/E of 1.40 compared to 1.15 with original PTC above $1 \mathrm{~cm}$. Elevated O/E of SPM was observed in MPTC with melanoma (radiation group 1.93, nonradiation group 1.69), prostate (radiation group 1.87, nonradiation group 1.50), kidney (radiation group 3.05 , nonradiation group 2.82), and lymphoma (radiation group 1.82, nonradiation group 1.23). With tumor size $>1 \mathrm{~cm}$, the incidence of lung cancer (0.68) was decreased in the population who did not undergo radiation treatment, but this was not replicated in patients who had a smaller tumor. Although the trend showed increased incidence of cancer in MPTC, the difference between groups did not reach a statistical significance.

3.4. Trends of SPM. Incidence of SPM at all sites was higher during 2000-2012 compared to 1992-1999 (O/E 1.24 versus 1.10) (Table 4). The incidence of the following SPMs increased from calendar period 1992-1999 to 2000-2012 (Table 4): all skin cancers $(\mathrm{O} / \mathrm{E} 1.48$ versus 1.22$)$, melanoma (1.47 versus 1.24 ), prostate cancer (1.41 versus 1.35 ), kidney cancer (2.71 versus 1.73), brain cancer (1.57 versus 0.94 ), and leukemia (1.91 versus 1.64). The difference between groups did not reach a statistical significance.

3.5. SPM among Gender. Risk of SPM was increased in both females and males (Table 4). During the period 2000-2012, $\mathrm{O} / \mathrm{E}$ of all cancers were higher in males compared to females (1.41 versus 1.17); a similar trend was observed during the calendar period 1992-1999 (1.16 versus 1.08) (Table 4). A higher incidence of all solid tumors (1.34 versus 1.18$)$, skin cancers including melanoma (1.68 versus 1.38 ), endocrine tumors (3.79 versus 1.23), non-Hodgkin lymphoma (1.65 versus 1.02), and leukemia (1.95 versus 1.88 ) was observed in males compared to females during the calendar period 2000-2012; a similar trend was observed during the period 1992-1999. Only breast cancer was increased in females compared to males (1.15 versus 0$)$. Females had a decreased risk of rectal cancer (0.54) and lung cancer (0.77), compared to males, during the period 1992-1999, but this finding was not observed in the period 2000-2012. The difference between groups did not reach a statistical significance.

3.6. Age of PTC and Subsequent Risk of SPM. There was an increased incidence of SPM among patients who were diagnosed with PTC at the younger age (Tables 5(a) and $5(\mathrm{~b}))$. O/E was the highest for patients whose PTC diagnosis was made at age 30-34 (O/E 1.43; 95\% CI 1.19-1.71) followed by age $35-39$ (O/E 1.30; 95\% CI; 1.13-1.49). O/E was elevated but was not statistically significant compared to reference population among age $0-29$. There was statistically significant elevated risk of salivary gland cancer in patients aged 5-29, peaking at age 5-9 (O/E 965.6; 95\% CI 24-5,380). There was also an increase in the incidence of leukemia and lymphoma peaking at age $30-34$ (O/E 2.27; 95\% CI 1.24-3.8). When combining effects of both radiation and age, there was increased incidence of leukemia, lymphoma, and salivary gland cancer in radioisotope group compared to nonradiation group at age $30-34$.

\section{Discussion}

To our knowledge, this is the most up-to-date US populationbased study to evaluate the risk for SPM among patients with PTC in SEER registry. We observed an increased SPM risk of many sites particularly salivary gland, bone, kidney, ureter, and hematologic malignancies. Interestingly, it showed decreased incidence of colorectal cancer especially pronounced in patients who did not undergo radiation therapy. Patients who underwent radioisotope therapy had the higher incidence of SPM, particularly bone, kidney, and hematologic malignancies. Males had a higher incidence of SPM than females. The incidence of SPM was higher after MPTC than PTC $>1 \mathrm{~cm}$, particularly in populations who underwent radiation therapy. We observed the higher incidence of SPM during the period 2000-2012 compared to the period 1992-1999. Patients who were diagnosed at younger age, particularly in their 30 s, had an increased risk of SPM. The results of this study are consistent with others [3-5, 8-10]. Radiation therapy including radioactive iodine therapy (RAI) is known to increase risk of SPM especially bone cancer, kidney cancer, hematologic malignancies, and prostate cancer in multiple studies including ours. This may be since RAI accumulates in bone marrow and is excreted through kidneys. Salivary gland and breast are known to express $\mathrm{Na}^{+} / \mathrm{I}^{-}$symporter which promotes selective uptake of RAI [26-28]. Given accumulation of these data, American Thyroid Association (ATA) revised a guideline to limit RAI on ATA high risk and selected ATA intermediate risk patients [29], excluding patients with unifocal tumors $<1 \mathrm{~cm}$ without other high risk features even in the presence of small-volume regional lymph node metastases. However, even though RAI use in MPTC has not been routinely recommended since 2009 , it has been reported that $38 \%$ of ATA low risk patients still undergo RAI [8]. Time trend analysis of radioactive iodine use in a cohort of 189,219 patients between 1990 and 2008 demonstrated a significant increase in the proportion of patients with thyroid cancer receiving radioactive iodine across all tumor sizes [30]. This may explain increasing trends of SPM over years. Our study results indicate MPTC patients, particularly ones who underwent radiation therapy, have higher incidence of SPM. This may be because patients with MPTC tend to be younger and thus more susceptible to adverse effects of radiation therapy along with longer life 


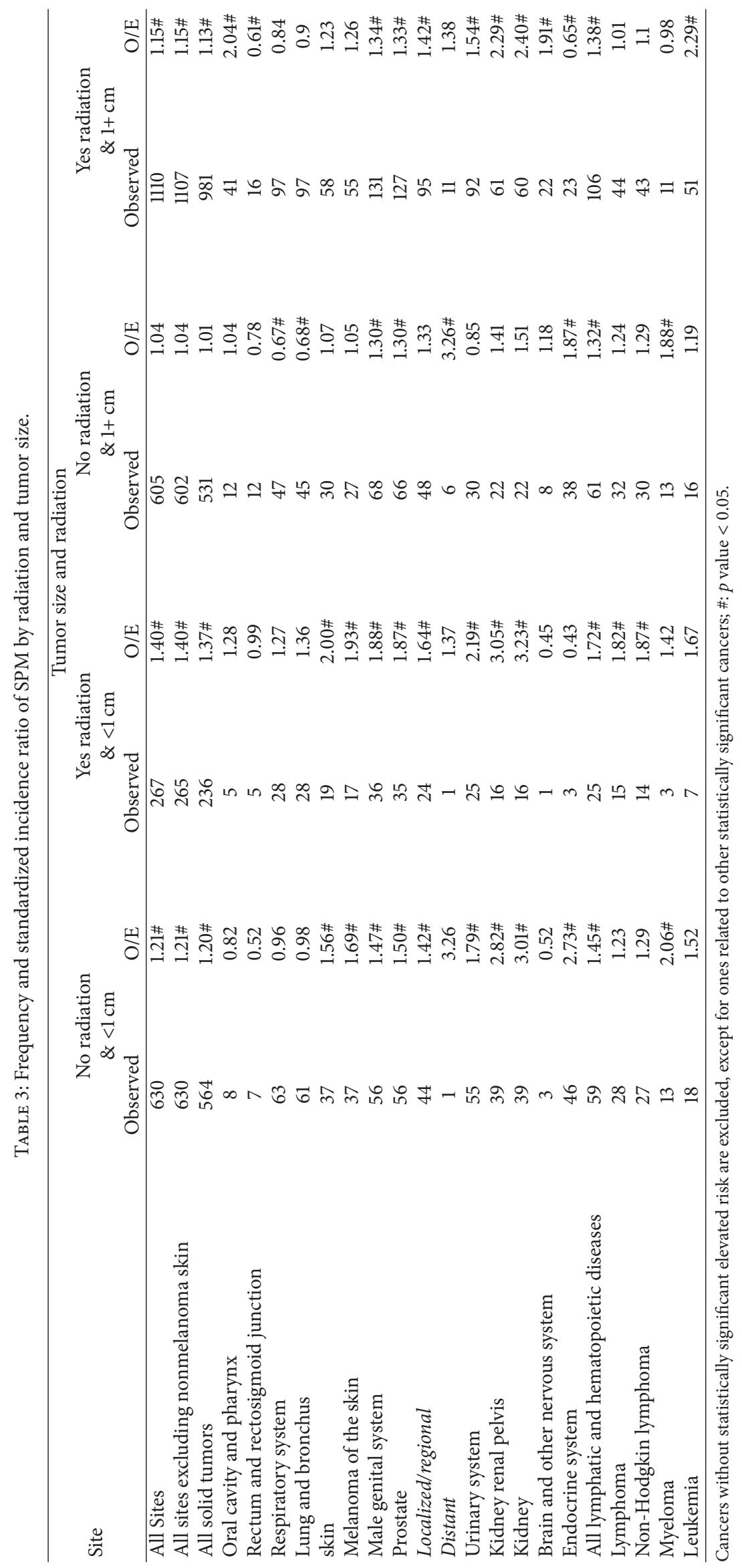




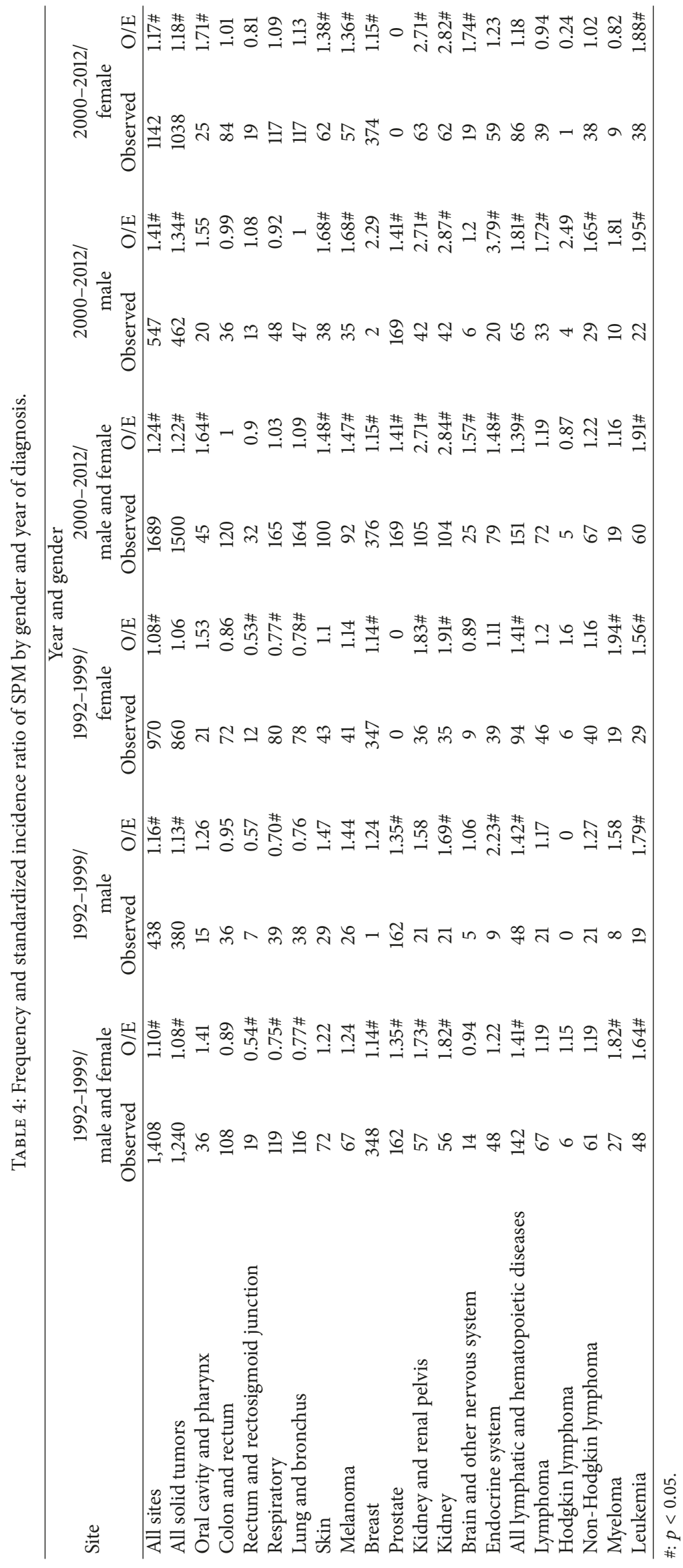




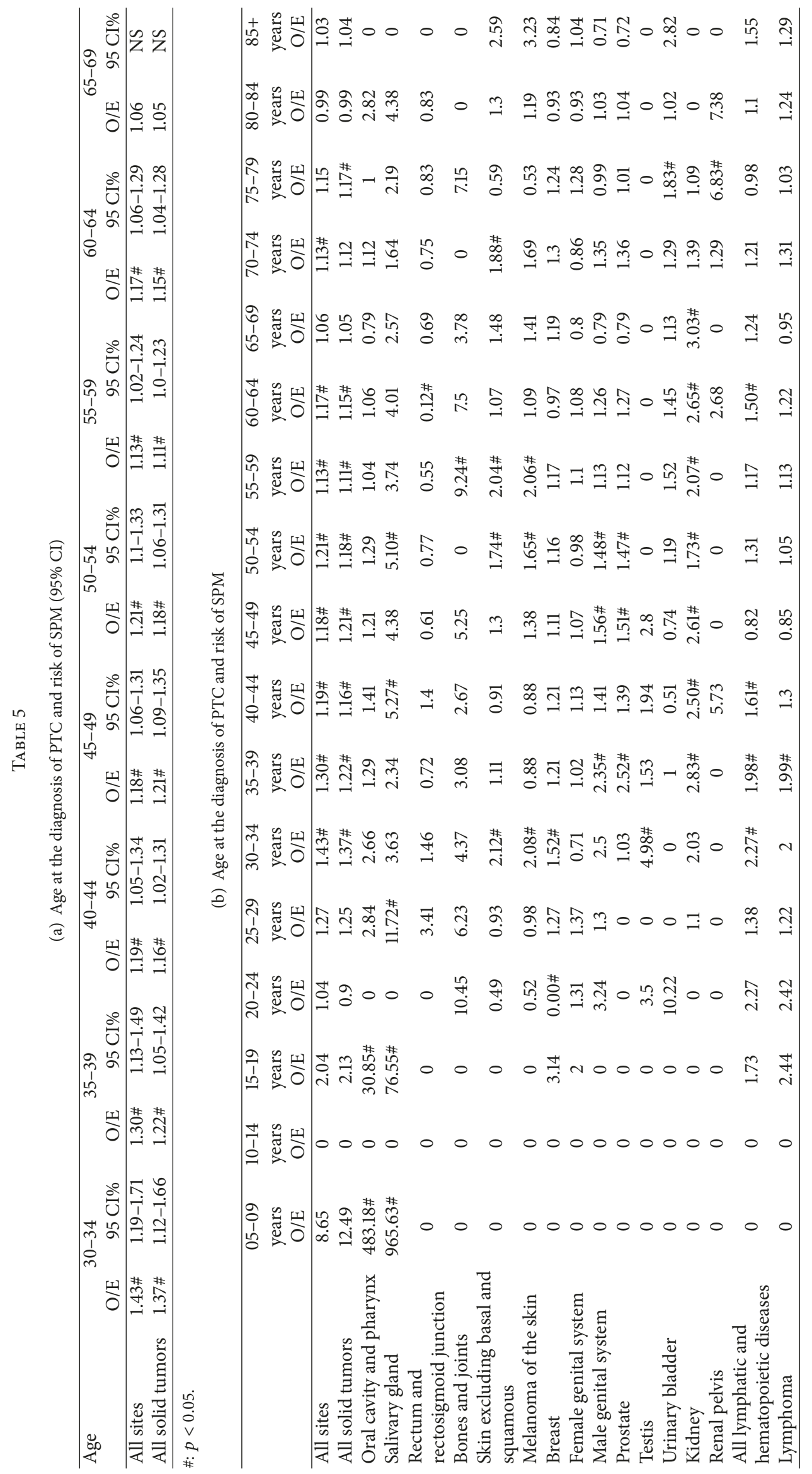




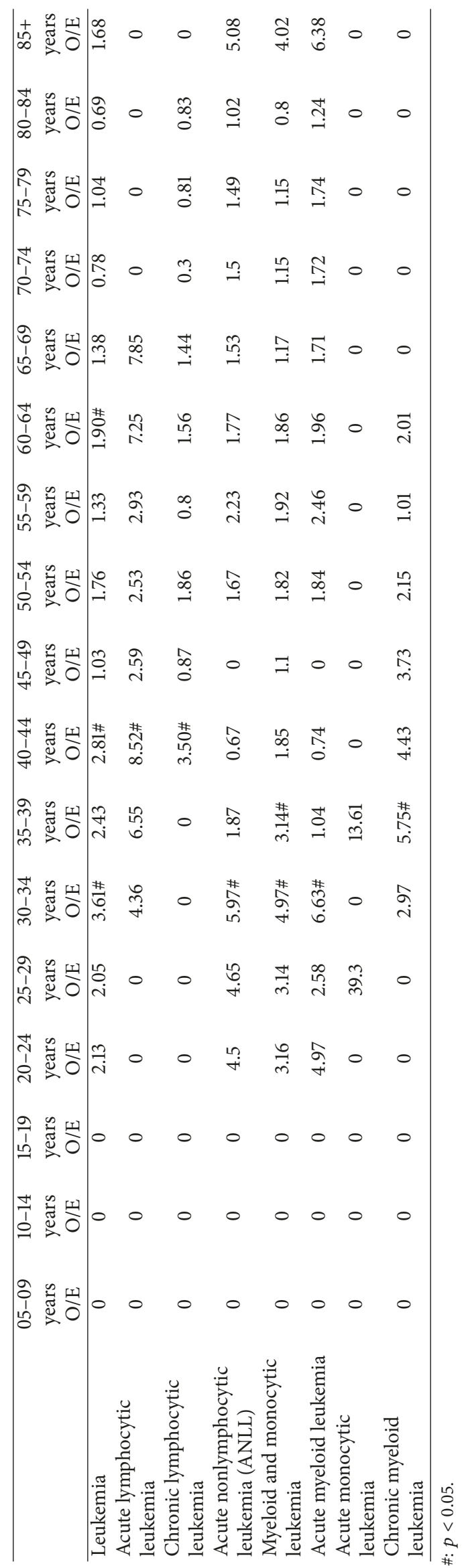


expectancy. In fact, our analysis shows higher incidence of SPM in patients who were diagnosed with PTC in younger age, with peak at ages 30-34. Our study, along with other studies, emphasizes that RAI should be used in selected population. This is especially the case since side effect profile of RAI has been underemphasized clinically compared to those of other radiation therapies such as external beam radiation therapy. Our results further indicate that males are more susceptible to SPM. Studies have shown that risk of SPM is higher in male for other forms of cancers such as colorectal, esophageal, and male breast cancer [31-33]. Male tends to have higher prevalence of cirrhosis and smoking history, which has been hypothesized to increased risks of certain cancers. However, risk of liver, lung, and squamous cell carcinoma was not higher in males compared to females in our cohort. More research is needed to explain higher incidence of SPM in males. Nonetheless, gender may be helpful to guide RAI use especially in ATA intermediate risk cases. Incidence of SPM at all sites was higher during 2000-2012 compared to 1992-1999. This most likely reflects prolonged latency period of tumor to develop in addition to high survival rate of PTC. Even though the overall incidence rate was lower, patients who did not undergo any RAI still had increased incidence of SPM. This may be due to genetic susceptibility of thyroid cancer patients. Studies suggest that the TERT mutation and germline mutations of FLCN are associated with both kidney cancer and PTC $[34,35]$. Mutations of CHEK2 are also associated with increased risk of kidney, thyroid, prostate, and breast cancers [36-39]. Recent advance in genomic diagnostics may enable tailoring screening strategies for patients with primary thyroid cancer for further risk of SPM. Our findings interestingly demonstrated decreased incidence of colorectal cancer in thyroid cancer survivors who did not undergo radiation therapy. There is an evidence that higher thyroid hormone level induces cell differentiation and mitigates tumor formation in colorectal cancer stem cells [40]. Since thyroid cancer survivors tend to be on TSH suppression therapy, hence they typically have higher thyroid hormone level than counterparts; this may unexpectedly lead to decreased incidence of colorectal cancer.

There are several limitations to our study. RAI administration is recorded reliably in the SEER program only in the adjuvant setting. Thus, RAI may not be recorded if this was given later for recurrent or new disease. The SEER program does not include information on RAI dosage. Hence, we were not able to analyze RAI dose and the risk of SPM. The increased diagnosis of prevalent malignancies may be an evidence of surveillance bias in our data. Patients who have been diagnosed with a previous malignancy may be more likely to seek routine and follow-up health care resulting in a perceived increase in SPM. Potential misclassification bias is also possible. However, given large size of the SEER cohort, any bias present is likely to be nondifferential.

The strength of this study is the use of a standardized, large, and well-established population database of the United States. In addition to the fact that the SEER program contains rich information allowing for robust analyses, this study included the most recent results available from SEER; this is particularly important, as a longer follow-up period for
SPM is optimal for analysis, given its potentially long latency period.

In summary, a large population-based tumor registry in the United States suggests an increased risk of SPM for all thyroid cancer survivors, particularly in survivors who received radiation therapy including RAI. Efficient monitoring strategies that include exposure to radiation, gender, and genetic susceptibility may successfully detect SPM earlier in the disease course. This is especially important given the excellent prognosis of the initial thyroid cancer itself.

\section{Conflicts of Interest}

The authors have no financial conflicts of interest to disclose.

\section{References}

[1] L. Davies and H. G. Welch, "Current thyroid cancer trends in the United States," JAMA Otolaryngology-Head \& Neck Surgery, vol. 140, no. 4, pp. 317-322, 2014.

[2] L. Rahib, B. D. Smith, R. Aizenberg, A. B. Rosenzweig, J. M. Fleshman, and L. M. Matrisian, "Projecting cancer incidence and deaths to 2030: the unexpected burden of thyroid, liver, and pancreas cancers in the United States," Cancer Research, vol. 74, no. 11, pp. 2913-2921, 2014.

[3] C. Kim, X. Bi, D. Pan et al., "The risk of second cancers after diagnosis of primary thyroid cancer is elevated in thyroid microcarcinomas," Thyroid, vol. 23, no. 5, pp. 575-582, 2013.

[4] C.-J. Teng, Y.-W. Hu, S.-C. Chen et al., "Use of Radioactive Iodine for Thyroid Cancer and Risk for Second Primary Malignancy: A Nationwide Population-Based Study," Journal of the National Cancer Institute, vol. 108, no. 2, Article ID djv314, 2016.

[5] J. L. Marti, K. S. Jain, and L. G. T. Morris, "Increased risk of second primary malignancy in pediatric and young adult patients treated with radioactive iodine for differentiated thyroid cancer," Thyroid, vol. 25, no. 6, pp. 681-687, 2015.

[6] S. Clement, L. Kremer, T. Links et al., "Is outcome of differentiated thyroid carcinoma influenced by tumor stage at diagnosis?" Cancer Treatment Reviews, vol. 41, no. 1, pp. 9-16, 2015.

[7] S. S. Patel and M. Goldfarb, "Well-differentiated thyroid carcinoma: The role of post-operative radioactive iodine administration," Journal of Surgical Oncology, vol. 107, no. 6, pp. 665-672, 2013.

[8] N. G. Iyer, L. G. T. Morris, R. M. Tuttle, A. R. Shaha, and I. Ganly, "Rising incidence of second cancers in patients with low-risk (T1N0) thyroid cancer who receive radioactive iodine therapy," Cancer, vol. 117, no. 19, pp. 4439-4446, 2011.

[9] A. P. Brown, J. Chen, Y. J. Hitchcock, A. Szabo, D. C. Shrieve, and J. D. Tward, "The risk of second primary malignancies up to three decades after the treatment of differentiated thyroid cancer," The Journal of Clinical Endocrinology \& Metabolism, vol. 93, no. 2, pp. 504-515, 2008.

[10] S. Subramanian, D. P. Goldstein, L. Parlea et al., "Second primary malignancy risk in thyroid cancer survivors: A systematic review and meta-analysis," Thyroid, vol. 17, no. 12, pp. 1277-1288, 2007.

[11] S. Yoshinaga, K. Mabuchi, A. J. Sigurdson, M. M. Doody, and E. Ron, "Cancer risks among radiologists and radiologic technologists: Review of epidemiologic studies," Radiology, vol. 233, no. 2, pp. 313-321, 2004. 
[12] G. M. Kendall, C. R. Muirhead, S. C. Darby, R. Doll, L. Arnold, and J. A. O'Hagan, "Epidemiological studies of UK test veterans: I. General description," Journal of Radiological Protection, vol. 24, no. 3, pp. 199-217, 2004.

[13] K. Hamatani, H. Eguchi, R. Ito et al., "RET/PTC rearrangements preferentially occurred in papillary thyroid cancer among atomic bomb survivors exposed to high radiation dose," Cancer Research, vol. 68, no. 17, pp. 7176-7182, 2008.

[14] L. B. Zablotska, E. A. Nadyrov, A. V. Rozhko et al., "Analysis of thyroid malignant pathologic findings identified during 3 rounds of screening (1997-2008) of a cohort of children and adolescents from Belarus exposed to radioiodines after the Chernobyl accident," Cancer, vol. 121, no. 3, pp. 457-466, 2015.

[15] N. Mitsutake, T. Fukushima, M. Matsuse et al., "BRAF V600E mutation is highly prevalent in thyroid carcinomas in the young population in Fukushima: A different oncogenic profile from Chernobyl," Scientific Reports, vol. 5, Article ID 16976, 2015.

[16] A. Kesminiene, A.-S. Evrard, V. K. Ivanov et al., "Risk of thyroid cancer among chernobyl liquidators," Journal of Radiation Research, vol. 178, no. 5, pp. 425-436, 2012.

[17] A. V. Brenner, M. D. Tronko, M. Hatch et al., "I-131 dose response for incident thyroid cancers in Ukraine related to the Chornobyl accident," Environmental Health Perspectives, vol. 119, no. 7, pp. 933-939, 2011.

[18] M. Takahashi, V. A. Saenko, T. I. Rogounovitch et al., “The FOXE1 locus is a major genetic determinant for radiationrelated thyroid carcinoma in Chernobyl," Human Molecular Genetics, vol. 19, no. 12, Article ID ddq123, pp. 2516-2523, 2010.

[19] E. D. Williams, "Chernobyl and thyroid cancer," Journal of Surgical Oncology, vol. 94, no. 8, pp. 670-677, 2006.

[20] Y. E. Nikiforov, "Radiation-induced thyroid cancer: What we have learned from chernobyl," Endocrine Pathology, vol. 17, no. 4, pp. 307-317, 2006.

[21] P. Jacob, T. I. Bogdanova, E. Buglova et al., "Thyroid cancer among Ukrainians and Belarusians who were children or adolescents at the time of the Chernobyl accident.," Journal of radiological protection : official journal of the Society for Radiological Protection, vol. 26, no. 1, pp. 51-67, 2006.

[22] A. But, P. Kurttio, S. Heinävaara, and A. Auvinen, "No increase in thyroid cancer among children and adolescents in Finland due to Chernobyl accident," European Journal of Cancer, vol. 42, no. 8, pp. 1167-1171, 2006.

[23] T. Ito, T. Seyama, K. S. Iwamoto et al., "Activated RET oncogene in thyroid cancers of children from areas contaminated by Chernobyl accident [1]," The Lancet, vol. 344, no. 8917, p. 259, 1994.

[24] B. S. Schoenberg and M. H. Myers, "Statistical methods for studying multiple primary malignant neoplasms," Cancer, vol. 40, no. 4 S, pp. 1892-1898, 1977.

[25] C. B. Begg, Z.-F. Zhang, M. Sun, H. W. Herr, and S. P. Schantz, "Methodology for evaluating the incidence of second primary cancers with application to smoking-relted cancers from the surveillance, epidmiology, and end results (SEER) program," American Journal of Epidemiology, vol. 142, no. 6, pp. 653-665, 1995.

[26] I. L. Wapnir, M. Van De Rijn, K. Nowels et al., "Immunohistochemical profile of the sodium/iodide symporter in thyroid, breast, and other carcinomas using high density tissue microarrays and conventional sections," The Journal of Clinical Endocrinology \& Metabolism, vol. 88, no. 4, pp. 1880-1888, 2003.
[27] O. Dohán, A. De La Vieja, V. Paroder et al., “The sodium/iodide symporter (NIS): characterization, regulation, and medical significance," Endocrine Reviews, vol. 24, no. 1, pp. 48-77, 2003.

[28] O. Dohán and N. Carrasco, "Advances in Na+/I- symporter (NIS) research in the thyroid and beyond," Molecular and Cellular Endocrinology, vol. 213, no. 1, pp. 59-70, 2003.

[29] B. R. Haugen, E. K. Alexander, and K. C. Bible, "2015 American Thyroid Association Management Guidelines for adult patients with thyroid nodules and differentiated thyroid cancer: The American Thyroid Association Guidelines Task Force on thyroid nodules and differentiated thyroid cancer," Thyroid, vol. 26, no. 1, pp. 1-133, 2016.

[30] M. R. Haymart, M. Banerjee, A. K. Stewart, R. J. Koenig, J. D. Birkmeyer, and J. J. Griggs, "Use of radioactive iodine for thyroid cancer," Journal of the American Medical Association, vol. 306, no. 7, pp. 721-728, 2011.

[31] S.-C. Chen, C.-J. Teng, Y.-W. Hu et al., "Secondary primary malignancy risk among patients with esophageal cancer in Taiwan: A nationwide population-based study," PLoS ONE, vol. 10, no. 1, Article ID e0116384, 2015.

[32] M.-H. Hung, C.-J. Liu, C.-J. Teng et al., "Risk of second nonbreast primary cancer in male and female breast cancer patients: A population-based cohort study," PLoS ONE, vol. 11, no. 2, Article ID e0148597, 2016.

[33] Y.-T. Lee, C.-J. Liu, Y.-W. Hu et al., "Incidence of second primary malignancies following colorectal cancer: A distinct pattern of occurrence between colon and rectal cancers and association of co-morbidity with second primary malignancies in a population-based cohort of 98,876 patients in Taiwan," Medicine (United States), vol. 94, no. 26, Article ID e1079, 2015.

[34] L. Dong, M. Gao, W.-J. Hao et al., "Case Report of Birt-HoggDubé Syndrome: Germline Mutations of FLCN Detected in Patients With Renal Cancer and Thyroid Cancer," Medicine, vol. 95, no. 22, p. e3695, 2016.

[35] R. J. A. Bell, H. T. Rube, A. Kreig et al., “The transcription factor GABP selectively binds and activates the mutant TERT promoter in cancer," Science, vol. 348, no. 6238, pp. 1036-1039, 2015.

[36] Cancer Genome Atlas Research N, "Integrated genomic characterization of papillary thyroid carcinoma," Cell, vol. 159, pp. 676-690, 2014.

[37] M. Siołek, C. Cybulski, D. Gąsior-Perczak et al., "CHEK2 mutations and the risk of papillary thyroid cancer," International Journal of Cancer, vol. 137, no. 3, pp. 548-552, 2016.

[38] C. Cybulski, T. Huzarski, B. Górski et al., "A novel founder CHEK2 mutation is associated with increased prostate cancer risk," Cancer Research, vol. 64, no. 8, pp. 2677-2679, 2004.

[39] C. Cybulski, B. Górski, and T. Huzarski, "CHEK2 is a multiorgan cancer susceptibility gene," American Journal of Human Genetics, vol. 75, no. 6, pp. 1131-1135, 2004.

[40] V. Catalano, M. Dentice, R. Ambrosio et al., "Activated thyroid hormone promotes differentiation and chemotherapeutic sensitization of colorectal cancer stem cells by regulating Wnt and BMP4 signaling," Cancer Research, vol. 76, no. 5, pp. 1237-1244, 2016. 


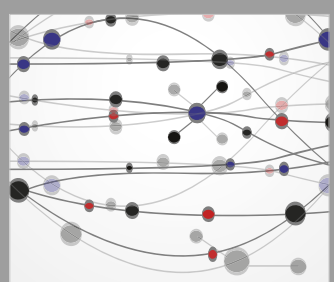

The Scientific World Journal
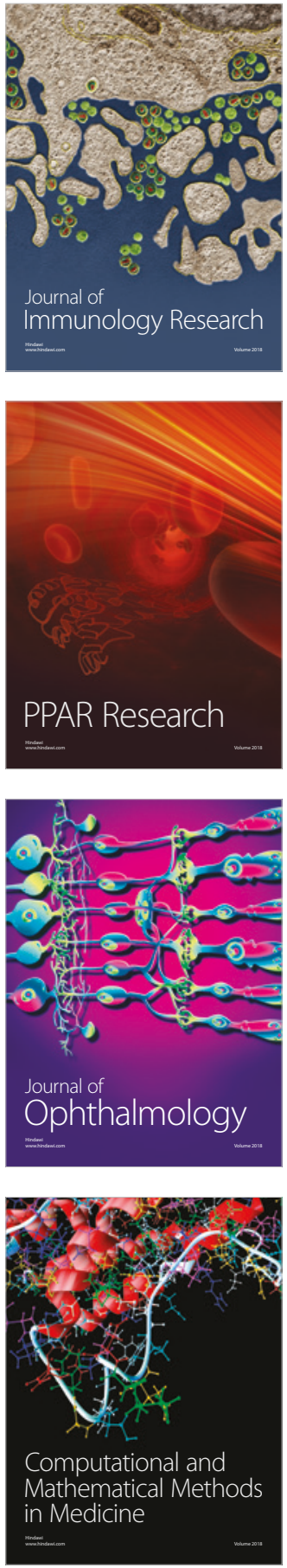

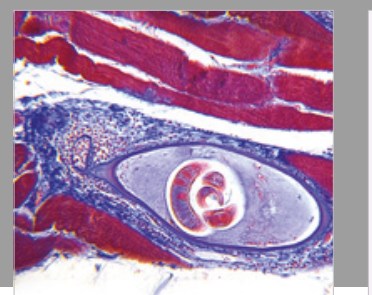

Gastroenterology Research and Practice

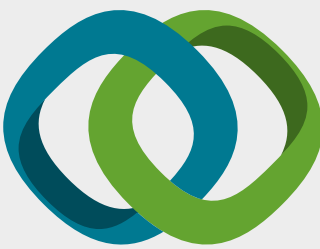

\section{Hindawi}

Submit your manuscripts at

www.hindawi.com
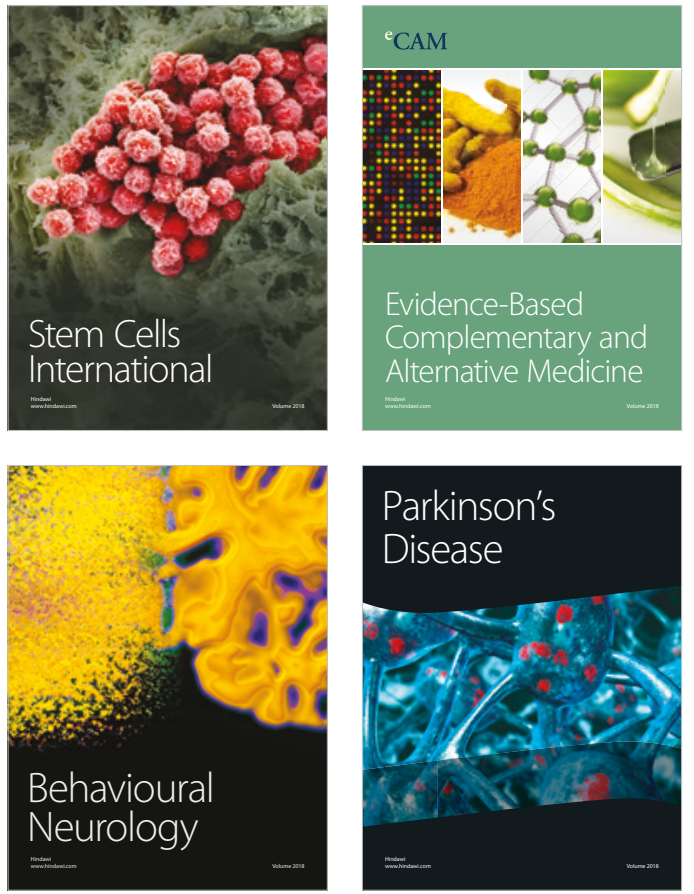

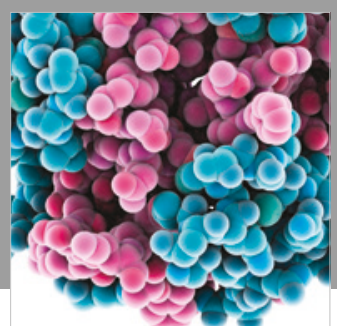

ournal of

Diabetes Research

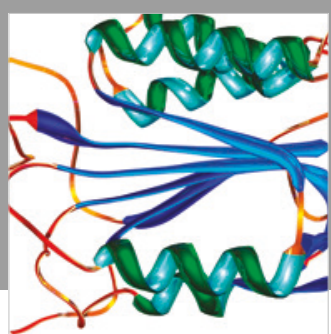

Disease Markers
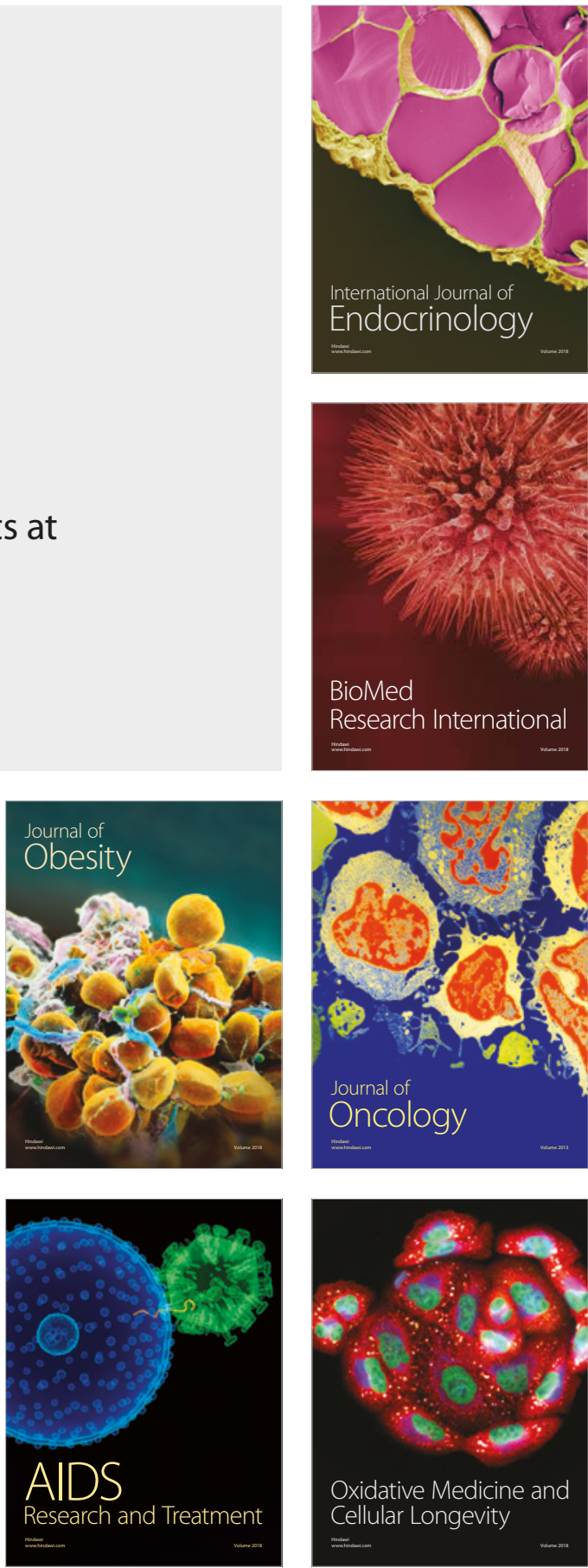